\title{
Directional sensitivity of MuSTAnG muon telescope
}

\author{
Grigori Karapetyan $^{1,2, *}$, Marina Ganeva ${ }^{1}$, and Rainer Hippler ${ }^{1}$ \\ 1 Institute of Physics, University of Greifswald, 17487 Greifswald, Germany \\ *Corresponding author: e-mail: grigori@yerphi.am \\ 2 Cosmic Ray Division, Yerevan Physics Institute, Yerevan 0036, Armenia
}

Received 26 November 2012 / Accepted 1 April 2013

\begin{abstract}
We investigate directional sensitivity of MuSTAnG muon telescope by deriving the distribution of secondary muons, which create the counting rate of telescope, by asymptotic directions of primary protons. This distribution, defined as "directivity function", allows us to clarify protons appearing from which direction essentially contribute to counting rate of detector. Directivity function has different behavior for the muons falling on the telescope at different zenith and polar angles. Vertical, West, and East fluxes exhibit strong maximums near the asymptotic longitude about $61^{\circ}$, whereas North and South fluxes have larger spread distributions. About $65 \%$ of muons, which create the Vertical counting rate of MuSTAnG, are produced by the primary protons, coming in the interval of asymptotic longitudes about $\left(50^{\circ}, 80^{\circ}\right)$. Using directivity function will allow one to more correctly determine the location of interplanetary disturbances. Analogous analysis, made for other muon detectors, will clarify their directional sensitivities, improving by this the forecasting capability of network of ground-based muon detectors.
\end{abstract}

Key words. space weather - coronal mass ejection (CME) - magnetosphere - cosmic ray - monitoring

\section{Introduction}

The primary cosmic rays reach the Earth after travelling through heliosphere. In times of CME propagation, the flux of cosmic rays is changed due to reflection from CME shock and magnetic clouds. It can lead to the change of proton flux near the Earth, consequently changing the flux of secondary muons in earth atmosphere. As a result, the counting rate of muon detector can vary depending on the presence of CME shocks and magnetic clouds. This phenomenon is a base for forecasting the arrival of CME shocks by monitoring counting rates of several muon detectors, located at different sites around the world (Munakata et al. 2000, 2001; Jansen et al. 2001; Leerungnavarat et al. 2003; Jansen \& Behrens 2008). These muon detectors create a global network of multidirectional muon detectors located at Nagoya (Japan), Hobart (Australia), and Sao Martinho (Brazil) and Greifswald (Germany). In this paper we investigate directional properties of Muon Spaceweather Telescope for Anisotropies Greifswald (MuSTAnG), located at the University of Greifswald.

At sea level the flux of secondary particles with energies $>150 \mathrm{MeV}$ consists mainly of muons. These muons are created via decay of pions, produced by the interaction of primary protons with air nuclei at the altitudes $\sim 15-20 \mathrm{~km}$. Since the muons lose energy on ionization of air atoms while propagating through the atmosphere, the counting rate of MuSTAnG is created by the muons with threshold energy (at production) of about $2.3 \mathrm{GeV}$.

For effective forecasting one needs to know from what direction outside of Earth magnetosphere have arrived primary protons, which are responsible for the counting rate of detector. Since Earth magnetic field declines the trajectory of protons, the direction of proton entering the magnetosphere is not coincident with the direction along which this proton falls on the top of atmosphere. The smaller is energy of proton the larger is the difference between these two directions.

For space science applications, one needs to know the direction of protons entering the magnetosphere - the asymptotic direction which is defined by asymptotic longitude $A$ and asymptotic latitude $B$. The standard analysis of asymptotic directions includes a relationship between the asymptotic direction and the kinetic energy $E$ of primary protons as functions $A(E)$ and $B(E)$. These functions can be derived by using computation code PLANETOCOSMICS (http://cosray.unibe.ch/ laurent/planetocosmics). However, this analysis is not sufficient to clarify the directions from where the main flux of protons comes.

Indeed, consider e.g., asymptotic directions of primary protons, which fall vertically on the top of atmosphere at Greifswald location (geographical coordinates $13^{\circ} 23^{\prime} \mathrm{E}, 54^{\circ} 05^{\prime} \mathrm{N}$, cutoff energy $\sim 2.5 \mathrm{GeV}$ for the vertical view direction). By getting the functions $A(E)$ and $B(E)$ from PLANETOCOSMICS one obtains the curve, presented in Figure 1.

It is seen that protons with energy about $400 \mathrm{GeV}$ come from direction in infinity close to vertical, however $\sim 20 \mathrm{GeV}$ protons decline in Earth magnetosphere on the angle about $45^{\circ}$, coming from asymptotic longitude about $60^{\circ}$. Protons with the energy about $3 \mathrm{GeV}$ decline larger, coming from the direction with asymptotic longitude about $170^{\circ}$, then asymptotic longitude for the $2.4 \mathrm{GeV}$ protons is about $-120^{\circ}$ and so on. All of these protons contribute to the vertical counting rate of MuSTAnG. However, this consideration does not clarify what protons give more contribution and what - less. Consequently it is difficult to locate the direction of primary protons, responsible for major part of counting rate. Thus analysis of asymptotic curves as presented in Figure 1 is not sufficient for determining the directions from where coming protons 


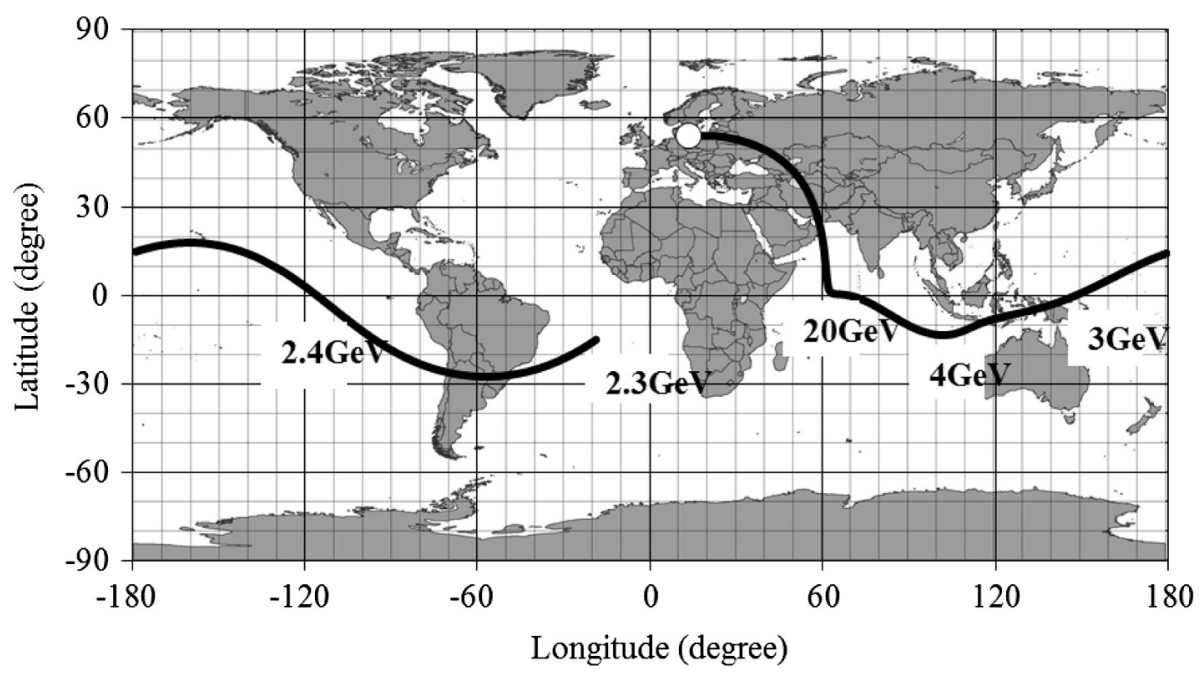

Fig. 1. Asymptotic directions (the solid line) of primary protons, falling vertically on the top of atmosphere above Greifswald location (open circle).

considerably contribute to counting rate of detector. For more advanced analysis one needs to know the distribution of protons, falling on the top of atmosphere by their asymptotic directions. The problem in such a form was first considered in McCracken (1962), Rao et al (1963), and then in Karapetyan (2010) for determining the acceptance cone of neutron monitors. The acceptance cone of a detector is the solid angle containing the asymptotic directions of approach of cosmic ray particles that significantly contribute to the counting rate of the detector (McCracken 1962).

In the present paper we consider this problem for muon detectors and derive the distribution of counting rate of detector by asymptotic directions of primary protons. This distribution, which is called below "directivity function", is a weighty characteristic of detector, determining its acceptance cone. The width and direction of acceptance cone of detector can be used in determination of the location of CME shock. If the acceptance cone of detector is narrow, then it can be used as well in studies of anisotropies, solar and sidereal variations.

\section{The MuSTAnG muon telescope}

The MuSTAnG is a multidirectional muon telescope. Figure 2 represents the schematic diagram of the MuSTAnG.

The muon telescope consists of upper detector layer ( 1 in Fig. 2), a lead layer of $5.1 \mathrm{~cm}$ thickness (2), and a lower detector layer (3). The distance between upper and lower detector layers is $0.95 \mathrm{~m}$. Every detector layer contains $4 \times 4=16$ detectors of $0.25 \mathrm{~cm}^{2}$ area. Thus the telescope contains 32 detectors in total and has full detection area of $4 \mathrm{~m}^{2}$. Detectors are arranged in detector units containing four detectors each. Inside of each detector unit there are located four scintillator plates of $5 \mathrm{~cm}$ thickness and $0.25 \mathrm{~cm}^{2}$ area. The scintillator plates are coupled via $1 \mathrm{~mm}$ diameter wavelength-shifting optical fibers to photomultiplier modules. Output pulses from the photo multiplier are discriminated and after suitable pulse-shaping, fed into the coincidence electronics. Thus the system comprises two layers each with 16 scintillators operating in between appropriate pairs. The directional information is derived from passage of muons through two, one upper and one lower, detectors. As a result the 1-min count rate data measured in the

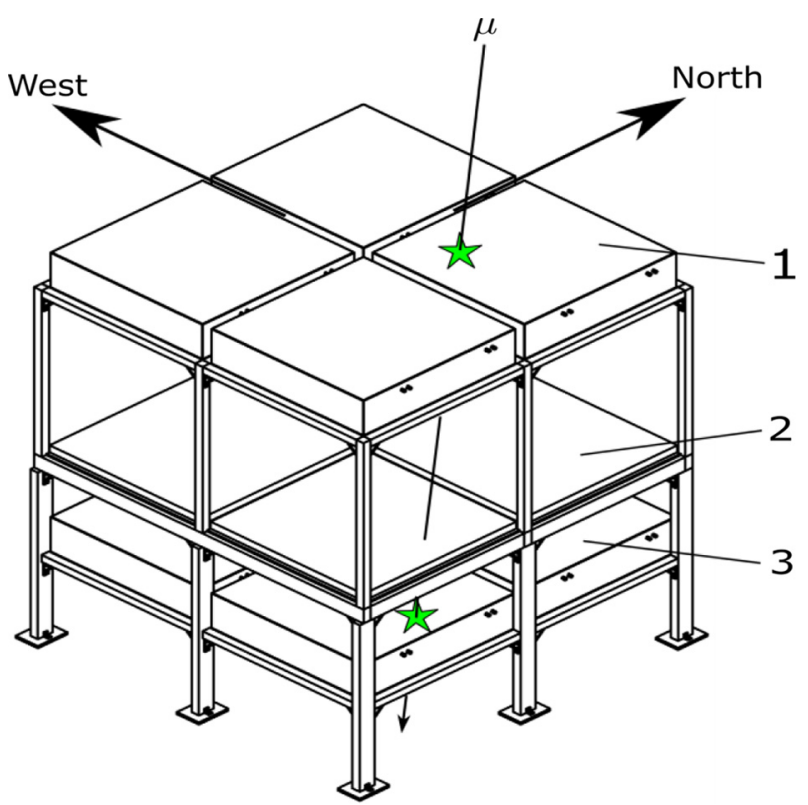

Fig. 2. Schematic diagram of MuSTAnG. $\mu$ indicates an incident muon. 1 is an upper detector level, 2 lead layer, 3 lower detector level.

vertical and four inclined view directions North, East, South, and West (Hippler et al. 2008). In the paper it is investigated how the count rates in these view directions are sensitive to asymptotic directions of primary protons.

\section{Directivity function of muon detector}

First we obtain the distribution by asymptotic directions of primary protons, falling on the top of atmosphere. Consider this distribution by asymptotic longitude $A$. The flux of primary protons is described by the known power-law spectral function $W(E) \sim(30 \mathrm{GeV} / \mathrm{E})^{2.7} 1 /\left(\mathrm{m}^{2} \mathrm{~s}\right.$ sr $\left.\mathrm{GeV}\right)$, where unit of energy is $\mathrm{GeV}$, and the dependence of asymptotic longitude A from the energy - function $A(E)$ is derived from PLANETOCOSMICS code. 


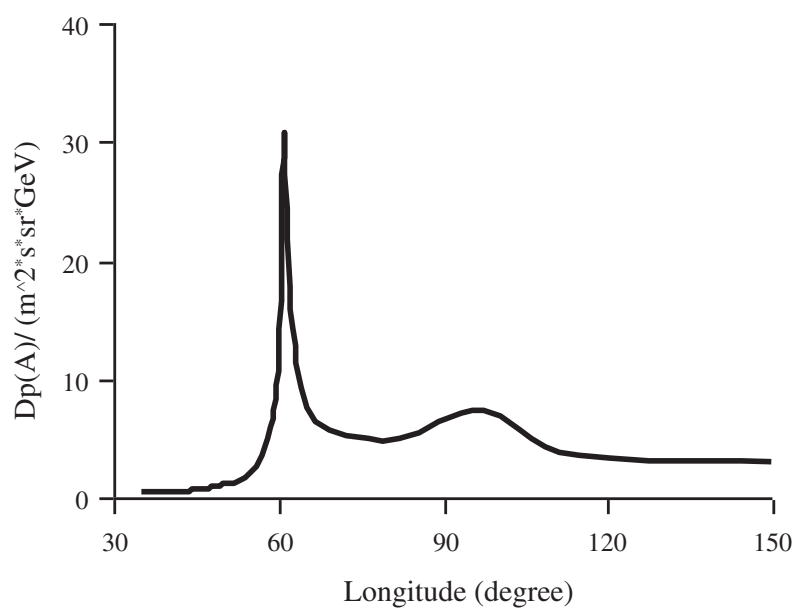

Fig. 3. Distribution of primary protons, falling vertically on the top of atmosphere above Greifswald by asymptotic longitudes.

By using the equality

$$
W(E) \mathrm{d} E=W(E(A))\left(\frac{\mathrm{d} E}{\mathrm{~d} A}\right) \mathrm{d} A
$$

the searched distribution by asymptotic longitude is determined by the function $D_{\mathrm{p}}(A)$, written as the following

$$
D_{\mathrm{p}}(A)=W(E(A))\left|\frac{\mathrm{d} A}{\mathrm{~d} E}\right|^{-1} .
$$

Thus, while the spectral function $W(E)$ represents the flux of protons versus their energy, the directivity function $D_{\mathrm{p}}(A)$ represents the same flux of proton versus their asymptotic longitude. It shows the contribution of primary protons coming from different asymptotic longitudes in the flux, falling on the top of atmosphere at the location to which the directivity function applies. Analogously directivity function versus the latitude $B$ can be derived. Since asymptotic directions are spread largely by the longitudes, we will investigate below directivity function versus asymptotic longitudes.

For vertically falling protons above Greifswald the function $D_{\mathrm{p}}(A)$ is shown in Figure 3, where we see a sharp maximum at the longitude $A=61.1^{\circ}$.

Thus the derived distribution $D_{\mathrm{p}}(A)$ reveals the presence of an asymptotic direction, from where incoming protons comprise a significant portion of the total flux of protons. However, $D_{\mathrm{p}}(A)$ does not yet clarify the main problem: the distribution of the counting rate by the longitude $A$ of primary protons. Since counting rate is created by the secondary muons, produced in atmosphere, the equivalent problem is the distribution of secondary muons by the longitude of primary protons. To solve this problem we will introduce a directivity function for secondary muons similar to (2) by replacement of the flux of protons $W(E)$ with the flux of muons produced by these protons, e.g., for the vertical view direction with the flux $V(E)$ defined below. Note that while $W(E)$ is differential flux of protons, nevertheless $V(E)$ is not the differential flux of muons. Really, differential flux of muons $S(x)$ is formed by all protons having energy larger than $x$, i.e., it is presented by the equation

$$
S(x)=\int_{x}^{\infty} y(E, x) W(E) \mathrm{d} E
$$

where yield function $y(E, x)$ connects the flux of muons $S(x)$, having energy $x$, with the flux of protons $W(E)$, having energy E.

At $E \sim x$ yield function $y(E, x)$ is negligibly small, therefore the value of integral (3) is determined by large values of $E$. For these $E$ the dependence of $y(E, x)$ from the energy of muons $x$ can be neglected, assuming that $y(E, x) \sim y(E)$ is the function of only the energy of proton. Then by differentiating (3) it is obtained

$$
y(E) \sim-\frac{1}{W(E)} \frac{\mathrm{d} S(E)}{\mathrm{d} E} .
$$

Integrating $y(E)$ in interval $\left(E_{\mathrm{th}}, E\right)$ in muon energy, where $E_{\text {th }} \sim 2.3 \mathrm{GeV}$, we obtain total yield function $Y(E)$, then by multiplying it on the proton flux $W(E)$, the searched flux of muons $V(E)$ for substituting in (2) is obtained

$$
\begin{aligned}
& Y(E)=\int_{E_{\mathrm{th}}}^{E} y(x) \mathrm{d} x, \\
& V(E)=Y(E) W(E) .
\end{aligned}
$$

Thus, the function $V(E)$ is the flux of muons with energies $\left(E_{\mathrm{th}} \ldots E\right)$, produced by the flux of protons $W(E)$. Functions $y(E), Y(E), W(E), S(E)$, and $V(E)$, calculated by (4)-(6), are presented in Figure 4. For $S(E)$ we prepared the function $8000 E /$ $(E+3)^{4.2} 1 /\left(\mathrm{m}^{2} \mathrm{~s} \mathrm{sr} \mathrm{GeV}\right)$ (where unit of energy is $\mathrm{GeV}$ ), which in the energy range from $\sim 1 \mathrm{GeV}$ to several hundred $\mathrm{GeVs}$ well interpolates experimental data, presented in (Bellotti et al 1999; Hebbeker \& Timmermans 2002; Yoshida et al 2006; Hagmann et al 2007).

It is seen that at energies $<10 \mathrm{GeV}$ the function $V(E)$ considerably decreases comparing with $W(E)$, because of ineffective production of muons by the protons with small energies. Thus substituting the flux of muons $V(E)$ from (6) to (2) instead of the flux of protons $W(E)$ we obtain the directivity function of muons $D_{\mathrm{m}}(A)$ as the following

$$
D_{m}(A)=V(E(A))\left|\frac{\mathrm{d} A}{\mathrm{~d} E}\right|^{-1} .
$$

Thus while spectral function $V(E)$ gives the distribution of muons by energies, the directivity function $D_{\mathrm{m}}(A)$ gives the distribution of these muons by asymptotic longitudes of primary protons. By integrating $V(E)$ over energies $>E_{\text {th }}$ we will obtain total flux of muons with energy $>E_{\mathrm{th}}$, the same value is obtained as well by integrating the differential spectrum of muons $S(E)$, i.e.,

$$
\int_{E_{\mathrm{th}}}^{\infty} V(E) \mathrm{d} E=\int_{E_{\mathrm{th}}}^{\infty} S(E) \mathrm{d} E \sim 55 /\left(\mathrm{m}^{2}, \mathrm{~s} \mathrm{sr} \mathrm{GeV}\right) .
$$

Function $D_{\mathrm{m}}(A)$ is shown in Figure 5. Compared to Figure 3 the maximum at the longitude about $61^{\circ}$ is seen sharper and values at larger longitudes are reduced more strongly. Such a decrease of muons, produced by the protons coming from larger longitudes, is conditioned by the reduction of function $V(E)$ at smaller energies, since corresponding protons larger decline in magnetosphere. From analysis of function $D_{\mathrm{m}}(A)$ it follows that 

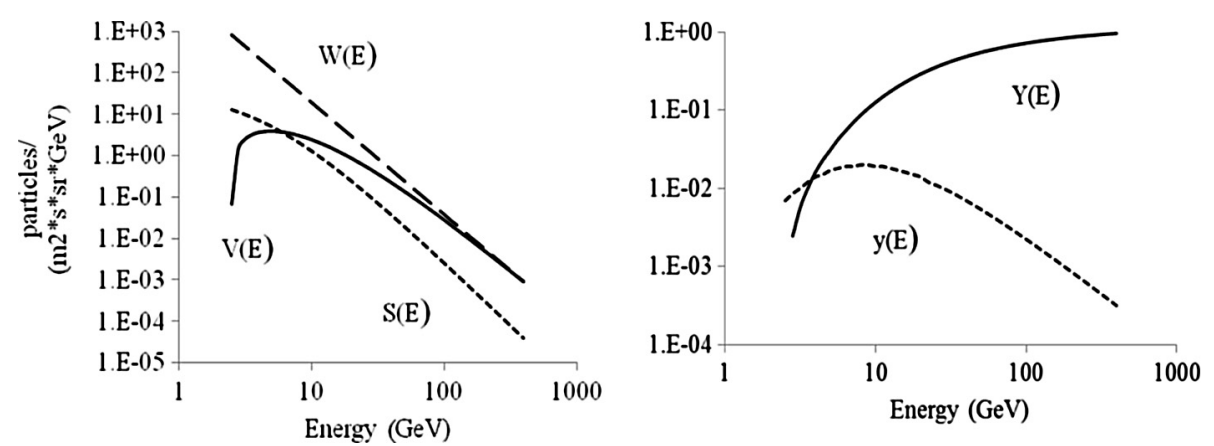

Fig. 4. Left panel: Differential spectums of protons $W(E)$ (the dashed line), muons $S(E)$ (the dotted line), and function $V(E)$ (the solid line). Right panel: yield functions $y(E)$ (the dotted line) and $Y(E)$ (the solid line).

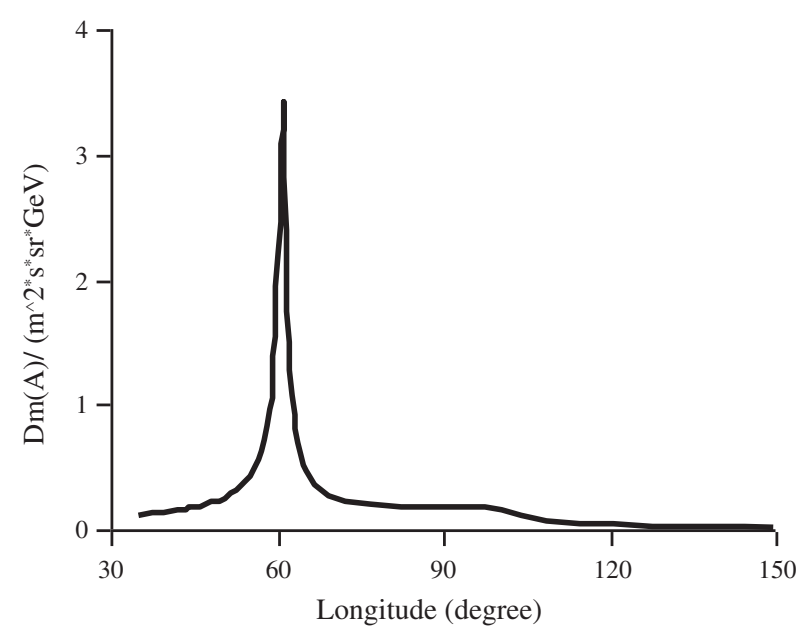

Fig. 5. Directivity function of vertical flux of muons, i.e., the distribution of muons, produced by vertically falling protons above Greifswald, by asymptotic longitudes of primary protons.

about $65 \%$ of all muons are produced by the protons coming from asymptotic longitudes in the interval $\left(50^{\circ}, 80^{\circ}\right)$. Corresponding asymptotic latitudes are spread in the interval $\sim\left(-10^{\circ}, 40^{\circ}\right)$. Thus, the vertical flux of secondary muons is highly sensitive to directions of primary protons, depending mainly on the protons coming from asymptotic longitudes in narrow interval $\sim 30^{\circ}$ around longitude $61^{\circ}$. These protons create the muons in energy interval $\sim(6 \mathrm{GeV}, 36 \mathrm{GeV})$, so that

$$
\frac{100 \int_{6}^{36} V(E) \mathrm{d} E}{\int_{0}^{\infty} V(E) \mathrm{d} E}=\frac{100 \int_{50}^{80} D_{\mathrm{m}}(A) \mathrm{d} A}{\int_{50}^{180} D_{\mathrm{m}}(A) \mathrm{d} A} \sim 65 \% .
$$

Thus the function $D_{\mathrm{m}}(A)$ is the distribution of vertically falling muons by asymptotic longitudes of primary protons, so it represents approximately the longitudinal sensitivity of Vertical count of MuSTAnG.

Analogously one can find the distribution functions of an inclined flux of muons. Consider inclined fluxes of muons, falling on the detector at zenith angle $30^{\circ}$ from four different directions: North, East, South, and West. Polar angle $\phi$ is counted clockwise from North direction, so that these directions have polar angles respectively $\phi=0,90^{\circ}, 180^{\circ}$, and $270^{\circ}$. These fluxes approximately represent directional sensitivity of corresponding inclined channels of MuSTAnG. Getting from PLANETOCOSMICS the functions $A(E)$ corresponding to these zenith and polar angles and calculating directivity functions
(7) we obtain the graphs, presented in Figure 6. It is seen that directivity functions for East and West fluxes of muons are approximately akin to that of Vertical flux, presented in Figure 5, whereas the North and South fluxes differ more from Vertical flux.

Note that the protons falling on the top of atmosphere at $30^{\circ}$ zenith angle have complicated trajectories in magnetosphere, so that protons at different energies and different asymptotic longitudes can arrive at the top of atmosphere with the same zenith and polar angles. It means that the function $D_{\mathrm{m}}(A)$, calculated by (7), can be multivalued in some interval of longitudes. In such cases all its branches should be summed to obtain real distribution function of muons.

Thus these directivity functions qualitatively represent directional sensitivity of North, East, South, and West counts of MuSTAnG. Based on these functions one can conclude that the most directional sensitivity have Vertical, East, and West counts, whereas the North and South counts are less sensitive to the asymptotic directions of primary protons. Therefore for accurate measurements one can use the sum of Vertical, East, and West counting rates. In Figure 7 there is presented crosssection of acceptance cone for Vertical count of MuSTAnG, inside which coming protons create about $65 \%$ of counting rate. Such presentation of directional sensitivity of detector by locating on the map appropriate area of cross-section of acceptance cone is more informative instead of usually used curves of asymptotic directions.

\section{Conclusions}

We investigated directional sensitivity of MuSTAnG muon telescope by deriving the distribution of counting rate by asymptotic directions of primary protons. This distribution, called directivity function, determines the acceptance cone of detector, showing protons appearing from which direction significantly contribute to counting rate. As a result the directional sensitivity of detector is shown on the map as the area of cross-section of acceptance cone. We have shown that a large contribution to counting rate is generated by protons, coming at narrow interval of longitudes $\sim\left(50^{\circ}, 80^{\circ}\right)$ and corresponding energies $\sim(36 \mathrm{GeV}, 6 \mathrm{GeV})$. The most sensitive measurements are the Vertical, East, and West counts of MuSTAnG, so that for accurate analysis the sum of these three counting rates should be used. The narrow directivity function of MuSTAnG will allow us to determine the location of CME shock more accurately, improving forecasting capability of the telescope. The method developed in this paper can be used for calculating directional sensitivity of other muon detectors, to clarify whether or not 
G. Karapetyan et al.: Directional sensitivity of MuSTAnG muon telescope
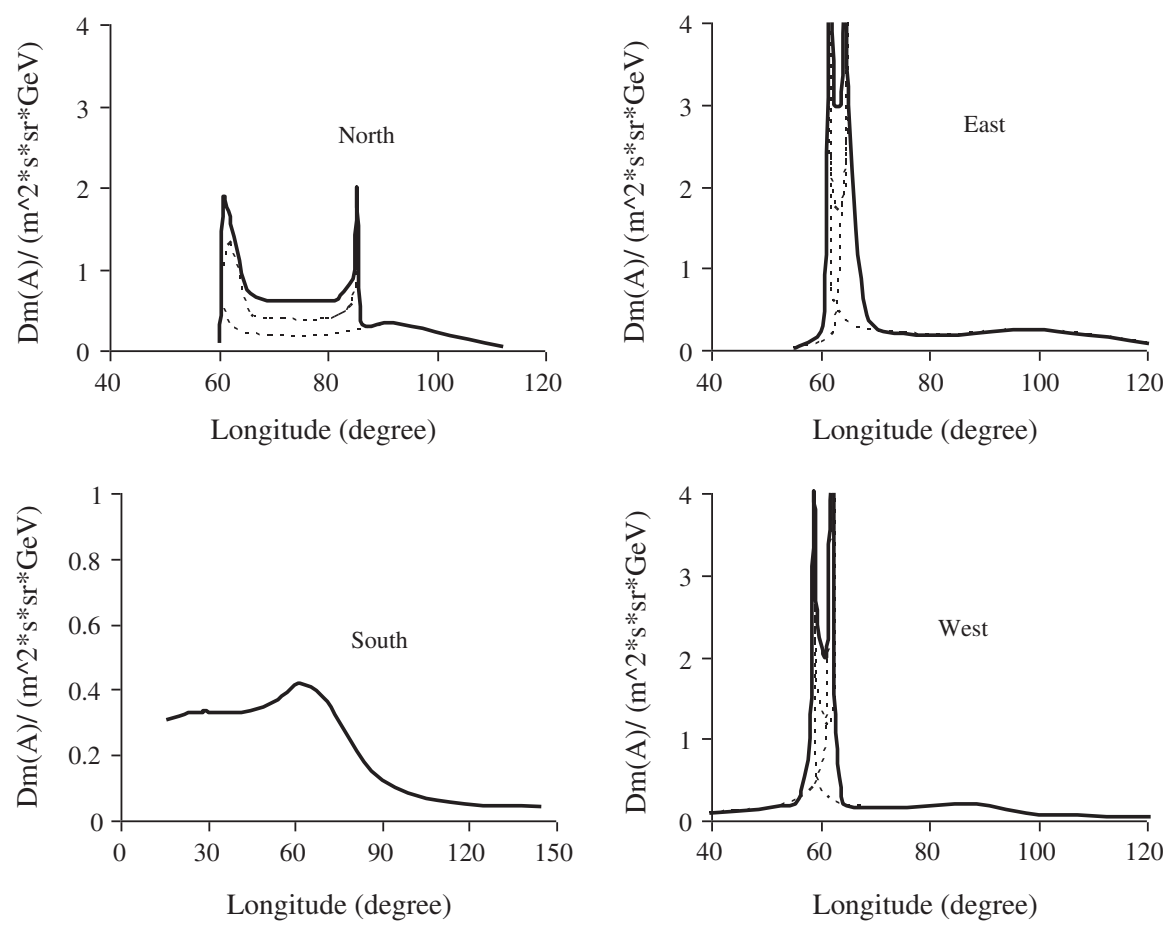

Fig. 6. Directivity functions of the inclined (at zenith angle $30^{\circ}$ ) fluxes of muons. All directivity functions, except of South, are multivalued, that are shown by the dotted curves. The net directivity functions, which are the sum of all branches of multivalued functions, are shown by the solid curves.

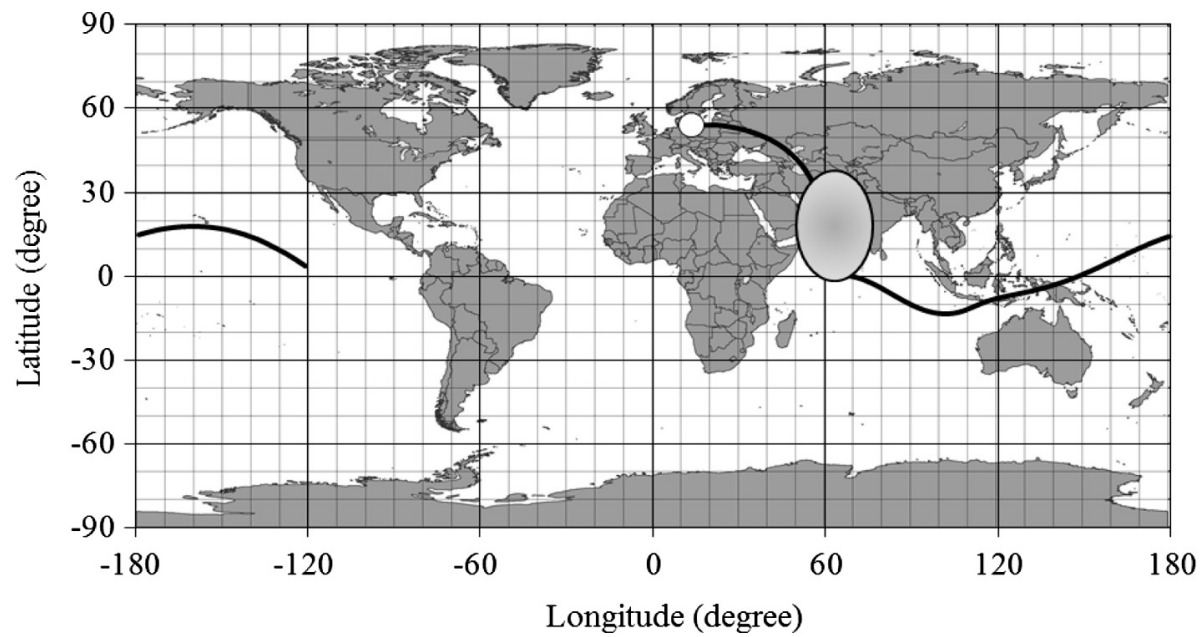

Fig. 7. The cross-section of the acceptance cone for vertical count of MuSTAnG, inside which incoming protons create about $65 \%$ of counting rate. The solid curve is asymptotic directions of vertical flux of protons.

their counting rates are also sensitive to asymptotic directions of primary protons. Muon detectors with narrow acceptance cones can be used as well for investigation of anisotropy of galactic cosmic rays by processing hourly counts, collected during several years.

Acknowledgements. The work was supported by the European Space Agency (ESA) and the Deutscher Akademischer Austauschdienst (DAAD). G.K. thanks DAAD for providing the opportunity for a research stay at the University of Greifswald.

\section{References}

Bellotti, R., F. Cafagna, M. Circella, and C.N. De Marzo, Balloon measurements of cosmicray muon spectra in the atmosphere along with those of primary protons and helium nuclei over midlatitude, Phys. Rev. D, 60, 052002, 1999.

Hagmann, C., D. Lange, and D. Wright, Monte Carlo Simulation of Proton-induced Cosmic-Ray Cascades in the Atmosphere, Lawrence Livermore National Laboratory, UCRL-TR, 2007.

Hebbeker, T., and C. Timmermans, A compilation of high energy atmospheric muon data at sea level, Astropart. Phys., 18, 107127, 2002.

Hippler, R., A. Mengel, F. Jansen, G. Bartling, W. Göhler, et al., First spaceweather observations at MuSTAnG - the muon spaceweather telescome for anisotropies at Greifswald, Proc. of 30th International Cosmic Ray Conference, Mexico, 1, pp. 347$350,2008$.

Jansen, F., K. Munakata, M.L. Duldig, and R. Hippler, Muon detectors - the real-time, ground based forecast of geomagnetic 
storms in Europe, ESA Space Weather Workshop, December, 2001.

Jansen, F., and J. Behrens, Cosmic rays and space situational awareness in Europe, 21st European Cosmic Ray Symposium in Kosice, Slovakia, pp. 626-632, 2008.

McCracken, K.G., The cosmic-ray flare effect: 1. Some new methods of analysis, J. Geophys. Res., 67, 423, DOI: 10.1029/JZ067i002p00423, 1962.

Karapetyan, G.G., Investigation of cosmic ray anisotropy based on Tsumeb neutron monitor data, Astropart. Phys., 33, 146-150, 2010.

Leerungnavarat, K., D. Ruffolo, and J.W. Bieber, Loss cone precursors to Forbush decreases and advance warning of space effects, Astrophys. J., 593, 587-596, 2003.
Munakata, K., J. Bieber, Y. Shin-ichi, C. Kato, M. Koyama, et al., Precursors of geomagnetic storms observed by the muon detector network, J. Geophys. Res., 105, 27457-27468, 2000.

Munakata, K., J. Bieber, J.W. Kuwabara, T. Hattori, K. Inoue, et al., A prototype muon detector network, covering a full range of cosmic ray pitch angles, Proc. 27th International Cosmic Ray Conference, Hamburg, 9, 3494-3497, 2001.

Rao, U.R., K.G. McCracken, and D. Venkatesan, Asymptotic cones of acceptance and their use in the study of the daily variation of cosmic radiation, J. Geophys. Res., 68, 345-369, 1963.

Yoshida, K., R. Ohmori, Y. Sato, T. Kobayashi, Y. Komori, et al., Cosmic-ray spectra of primary protons and high altitude muons deconvolved from observed atmospheric gamma rays, Phys. Rev. D, 74, 083511, http://cosray.unibe.ch/ laurent/planetocosmics, 2006.

Cite this article as: Karapetyan G, Ganeva M \& Hippler R: Directional sensitivity of MuSTAnG muon telescope. J. Space Weather Space Clim., 2013, 3, A16. 